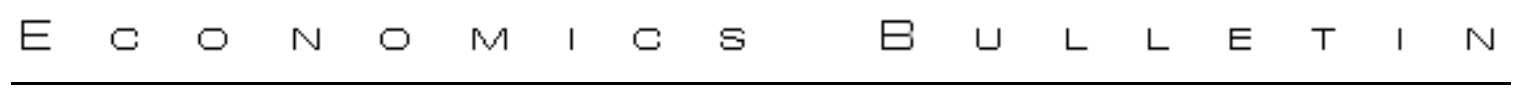

\title{
Indeterminacy and the distribution of growth rates
}

\author{
Greiner Alfred \\ University of Bielefeld, Department of Economics
}

\author{
Krueger Jens J. \\ Friedrich-Schiller-University Jena, Department of \\ Economics
}

\begin{abstract}
In endogenous growth theory models exist which are characterized by local and global indeterminacy. These concepts imply that economies differ with respect to their growth rates on the transition path (local indeterminacy) as well as their long-run growth rates (global indeterminacy). While the empirical density function of the levels of aggregate GDP has been the subject of a great many studies, the distribution of the growth rates has not yet been analyzed. In this paper, recent research on the evolution of the world income distribution is expanded by an analysis of the evolution of the distribution of growth rates for a sample of 104 countries. It is found that this distribution is remarkably stable over the period 1960-90.
\end{abstract}

Citation: Alfred, Greiner and Krueger Jens J., (2001) "Indeterminacy and the distribution of growth rates." Economics Bulletin, Vol. 15, No. 5 pp. 1-7

Submitted: October 1, 2001. Accepted: November 21, 2001.

URL: http://www.economicsbulletin.com/2001/volume15/EB-01040002A.pdf 


\section{Introduction}

In endogenous growth theory models exist which reveal local and/or global indeterminacy. Local indeterminacy means that different economies converge to the same balanced growth path (BGP) in the long-run. The growth rates of economic variables, however, differ as long as the economies are on the transition path. Global indeterminacy implies that there are several BGPs to which an economy may converge in the long-run. Examples of such models which show both local and global indeterminacy are Benhabib and Farmer (1994), Benhabib and Perli (1994), Benhabib, Perli and Xie (1994), Greiner and Semmler (1996) and RaurichPuigdevall (2000).

Nevertheless, as far as we know, the empirical distribution of growth rates has not yet been studied empirically. However, this is not the case for the levels of per capita GDP. Recent work of Bianchi (1997), Jones (1997), Paap and van Dijk (1998) and most notably Quah (1993a,b; 1996a,b; 1997) investigates the shape of the distribution of suitably transformed real GDP per worker or GDP per capita data and traces the changes of the shape of this distribution over time. The central finding of these papers is an evolution of the world income distribution towards a bimodal shape with one mode in the range of low relative income and a second mode in the range of high relative income. This finding has been considered as a new stylized fact in modern growth theory (see Durlauf and Quah, 1999).

In contrast to that, this paper provides some preliminary evidence about the question of whether there are similarities in the evolution of the relative income distribution and in the evolution of the corresponding growth rates. Therefore, we study the distribution of annual growth rates of real GDP per worker instead of the corresponding levels. The central result of this largely descripitve analysis is a remarkably stable shape of the distribution of growth rates over the period 1960-90 for a sample of 104 countries.

The rest of the paper is organized as follows. In the next section we give a brief introduction to the concept of local and global indeterminacy. In section 3, we present results that concern the empirical distribution of growth rates and section 4 concludes the paper.

\section{Non-Uniqueness of Growth Rates in Endogenous Growth Models}

Typically, the starting point in endogenous growth models is an intertemporal optimization problem of a representative individual, who maximizes a discounted stream of utility arising from consumption and/or leisure over an infinite time horizon, subject to a budget constraint. The individual produces the goods he consumes by himself or he receives labour and capital income from the productive sector. In the latter case, the productive sector has to be explicitly modelled where it is often assumed that the economy is characterized by completely competitive markets. This implies that the wage rate equals the marginal product of labour and the interest rate equals the marginal product of capital. 
Solving the intertemporal optimization problem of the individual and imposing equilibrium conditions gives a system of autonomous differential equations which completely describes the dynamics of the economy. The differential equations are given by ${ }^{1}$

$$
\begin{aligned}
& \dot{k}=f(k, c), k(0)=k_{0} \\
& \dot{c}=g(k, c)
\end{aligned}
$$

with $k \in \mathbb{R}_{+}^{n}$ a $n$-dimensional vector of capital goods with given initial values $k_{0}$ and $c \in \mathbb{R}_{+}^{m}$ a $m$-dimensional vector of control variables like consumption or leisure for example. $f: \mathbb{R}_{+}^{n} \times \mathbb{R}_{+}^{m} \rightarrow \mathbb{R}^{n}$ and $g: \mathbb{R}_{+}^{n} \times \mathbb{R}_{+}^{m} \rightarrow \mathbb{R}^{m}$ are $C^{1}$ vectorial functions.

Endogenous growth arises if the marginal product of capital does not converge to zero as capital rises. From an economic point of view this is feasible if there are for example positive externalities of capital which have a stimulating effect on the marginal product of capital. Other economic factors which may lead to endogenous growth are the formation of human capital or of a stock of knowledge. In case of endogenous growth the right hand sides of equations (1) and (2) do not have a rest point implying that there is positive growth in the long-run.

A standard practise to study the dynamics of endogenous growth models is to perform a change of variables such that a new dynamic system results and such that a rest point of this new system gives a BGP on which all variables grow at constant rates. Defining new variables $x_{i}=x_{i}(k, c), i=1, \ldots, m+n-1, C^{1}$ functions of the original state and control variables the dynamics around a BGP are described by

$$
\dot{x}_{i}=h_{i}\left(x_{1}, \ldots, x_{m+n-1}\right), i=1, \ldots, m+n-1
$$

A simple example for such transformations are ratios of endogenous variables, like the ratio of consumption to physical capital or the ratio of consumption to the stock of knowledge.

Analyzing system (3) demonstrates that endogenous growth models may be characterized by both local and global indeterminacy. Local indeterminacy implies the following. Assume that there exists a rest point $x_{i}^{*}$ of (3) and that this point is completely stable in the sense that all trajectories satisfying (3) which start in the neighbourhood of $x_{i}^{*}$ will converge to this rest point. Then there exists a continuum of equilibrium paths which converge to the rest point. This holds because only the capital stocks are fixed at time $t=0$ while the controls, such as consumption, can be chosen freely. Consequently, there also exists a continuum of transitional growth rates since these are affected by the choice of the initial controls. In the long run, however, the economies all grow at the same rate since they converge to the same rest point $x_{i}^{*}$ . Which level the control variables take at $t=0$ depends on non-economic factors like cultural or institutional ones for example.

Global indeterminacy refers to the long-run behaviour of the system and means the following. If (3) is characterized by multiple steady states then there exist multiple BGPs and,

\footnotetext{
${ }^{1}$ We omit the argument $t$ since no ambiguity arises.
} 
consequently, multiple balanced growth rates. Which one of these is approached by the economy again depends on the initial level of the control variables. In this case we speak of global indeterminacy.

Both local and global indeterminacy imply non-unique growth rates. $^{2}$ In the first case, the transitional growth rates will be different for different countries. In the second case, the longrun balanced growth rates are expected to differ. If these phenomena can be observed in reality one would expect a distribution of growth rates which is not unimodal. In the next section we therefore take a look at the empirical distribution of growth rates.

\section{The Empirical Distribution of Growth Rates}

The main tool used to get a visual impression of distribution dynamics is kernel density estimation for one dimensional data (see Wand and Jones, 1995, ch. 2). We apply the kernel density estimator with a biweight kernel function and a bandwidth parameter selected according to the rule of thumb proposed by Silverman (1986, pp. 47f.). Using this estimator with respect to annual growth rates of real GDP per worker for 104 countries gives the series of density plots for the years $1960-90$ that is shown in figure $1 .^{3}$

Figure 1

Estimated Growth Rate Distribution

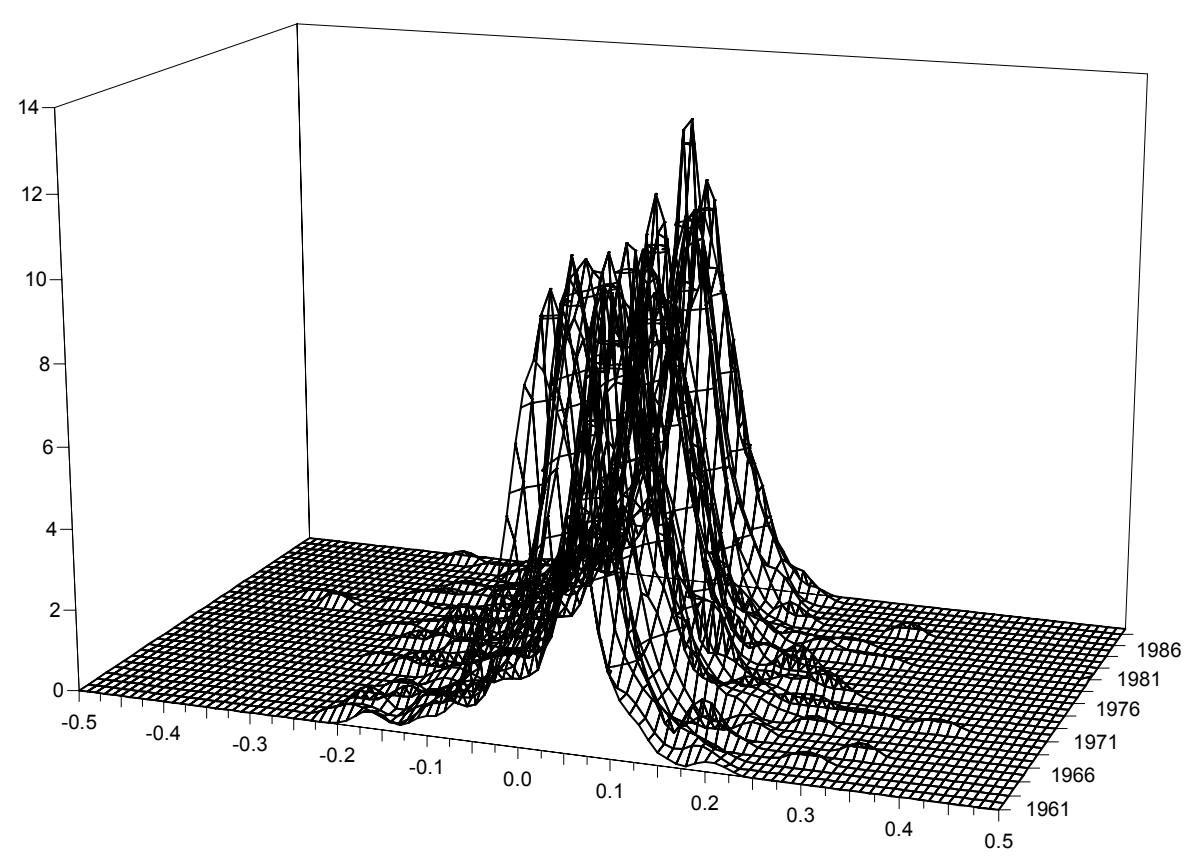

\footnotetext{
2 It should be noted that local and global indeterminacy are not the only source of multiple equilibria. Other sources are e.g. sunspots based on self-fulfilling prophecies.

3 The data are taken from the Penn World Table 5.6 (see Summers and Heston, 1991).
} 
In addition, figure 2 presents four single density profiles for the years 1961, 1970, 1980 and 1990 which are simply cuts through the plot of figure 1 . Both figures strongly suggest that the distribution of growth rates is persistently unimodal and further that its shape is remarkably stable over time with respect to both its mode and dispersion.

Figure 2

Growth Rate Density Profiles for Selected Years

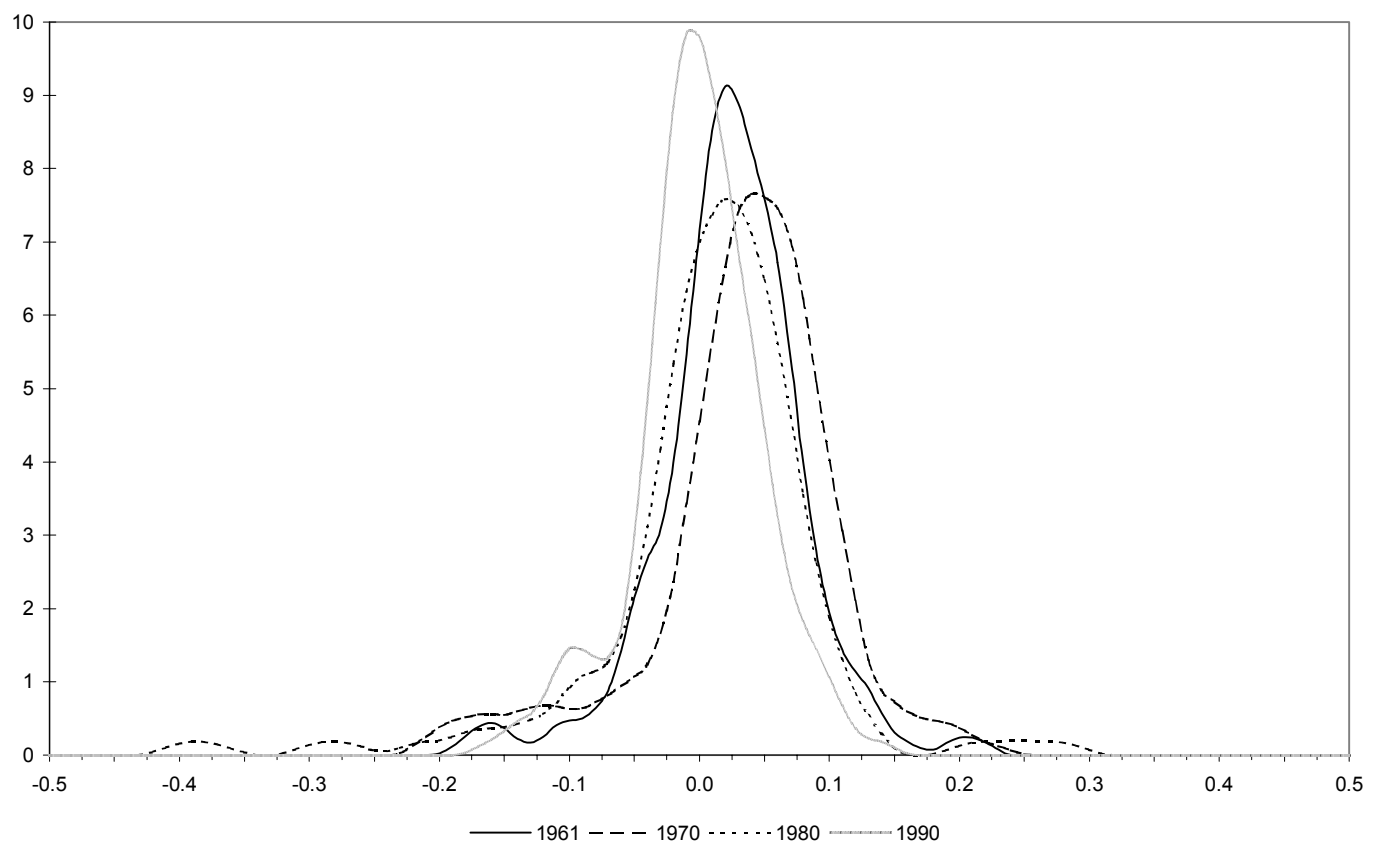

This impression of stability over time is further sharpened by looking at the dynamics of estimates of the first, second and third moments of the distribution of growth rates as given in figure 3. Here we observe a declining mean and median over the whole sample period. Both reach a value very close to zero for the mean and median growth rates at the end of the sample period. The world wide impact of the two oil price shocs in the middle of the 1970s and the beginning of the 1980s is clearly visible. The standard deviation can also be regarded as stable in that it shows some fluctuations around a constant value. A related statement applies to the skewness which seems also to be stationary around a value of approximately zero with some marked deviations during the 1980s, indicating a by and large symmetric growth rate distribution. 
Figure 3

Empirical Moments of the Growth Rate Distribution

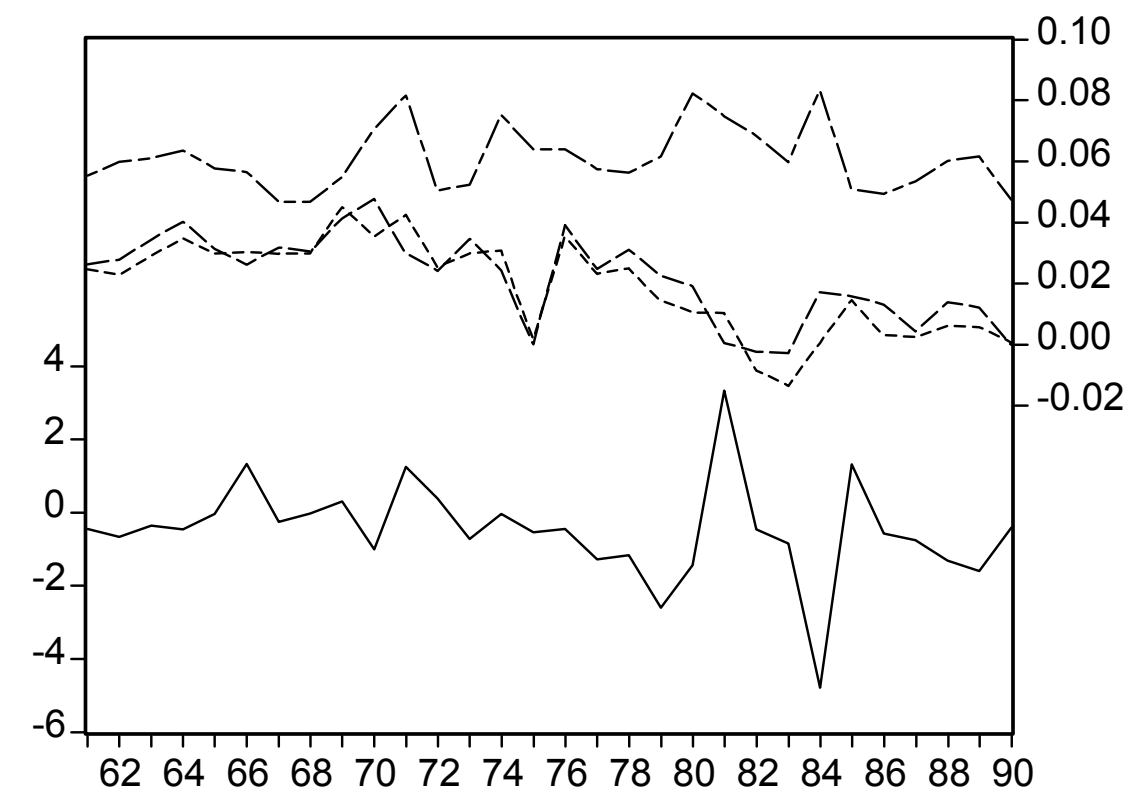

SKEWNESS

MEAN

MEDIAN

STDDEV

Table 1

Unit Root Tests

\begin{tabular}{lcccc}
\hline Test & Mean & Median & Std. Deviation & Skewness \\
\hline $\operatorname{ADF}(1)$ & -1.1054 & -1.6265 & $-3.7525^{* * *}$ & $-4.0580^{* * *}$ \\
$\operatorname{ADF}(2)$ & -0.8655 & -1.2550 & $-2.7956^{*}$ & $-4.0839 * * *$ \\
$\operatorname{PP}(1)$ & -1.7483 & -2.2216 & $-4.0626^{* * *}$ & $-5.5308^{* * *}$ \\
$\operatorname{PP}(2)$ & -1.7663 & -2.1986 & $-3.9860^{* * *}$ & $-5.5447 * * *$ \\
\hline
\end{tabular}

Note: ADF and PP indicate the augmented Dickey-Fuller and the Phillips-Perron t test for unit roots with 1 and 2 lags, respectively; the specification includes an intercept but no trend; significant rejection of the unit root null is indicated by $*$ on $10 \%, * *$ on $5 \%$ and $* * *$ on $1 \%$ level.

In addition, we perform unit root tests with respect to the time series of the moments to obtain a more formal confirmation for the results of the visual inspection of the figures. The augmented Dickey-Fuller and Phillips-Perron t test statistics (see Maddala and Kim, 1998, ch. 3) are summarized in table 1 . The chosen lag lengths of one and two periods should be sufficient to remove serial correlation in the present case of yearly data. The results give strong support for 
the stationarity of the standard deviation and the skewness series, whereas the unit root null of the mean and median series cannot be rejected at conventional significance levels. The nonstationarity of the mean and median series is clearly a consequence of the declining trend which is also visible in figure 3 . This trend need not necessarily be stochastic which is confirmed by the fact that the rejection of the unit roots in both cases becomes somewhat stronger if a linear deterministic trend is included in the test regression in addition to the intercept.

\section{Conclusion}

The empirical part of the paper has demonstrated that there is a persistently unimodal distribution of growth rates of GDP per worker with a declining mean growth rate and with stationary second and third moments. Therefore, on average, different countries have the same growth rate of GDP per worker. As concerns the theoretical part of this contribution this implies that endogenous growth models featuring indeterminacy are not correct if they are used to describe the process of economic growth in a cross section of countries on average. This holds because such models are characterized by multiple growth paths predicting that the empirical distribution of growth rates should not be unimodal. Nevertheless, one should not reject such models as irrelvant from an empirical point of view since they can help to explain different growth performances of single countries. A typical example where growth models with indeterminacy may be helpful are the Philippines and South Korea. As pointed out by Lucas (1993) these two countries basically had the same technology and preferences and were also characterized by almost identical initial conditions with respect to capital stocks in 1960. But, inspite of that, subsequent growth rates have been quite different over the last decades. So our evidence against the validity of growth models featuring indeterminacy can only be taken as preliminary and further research focusing on intra-distribution dynamics is required.

\section{References}

Benhabib, J., and R.E.A. Farmer (1994), "Indeterminacy and Increasing Returns", Journal of Economic Theory 63, 19-41.

Benhabib, J., and R. Perli (1994), "Uniqueness and Indeterminacy", Journal of Economic Theory 63, 113-142.

Benhabib, J., R. Perli, and D. Xie (1994), "Monopolistic Competition, Indeterminacy and Growth", Ricerche Economiche 48, 279-298.

Bianchi, M. (1997), "Testing for Convergence: Evidence from Non-Parametric Multimodality Tests", Journal of Applied Econometrics 12, 393-409.

Durlauf, S.N., and D. Quah (1999), "The New Empirics of Economic Growth", in: J.B. Taylor, and M. Woodford (eds.), Handbook of Macroeconomics, vol. 1A, Amsterdam: Elsevier, 235-308. 
Greiner, A., and W. Semmler (1996), "Multiple Steady States, Indeterminacy, and Cycles in a Basic Model of Endogenous Growth", Journal of Economics 63, 79-99.

Jones, C.I. (1997), "On the Evolution of the World Income Distribution", Journal of Economic Perspectives 11, 19-36.

Lucas, R.E. (1993), "Making a Miracle", Econometrica 61, 251-272.

Maddala, G.S., and I.-M. Kim (1998), Unit Roots, Cointegration, and Structural Change, Cambrigde (Mass.): Cambridge University Press.

Paap, R., and H.K. van Dijk (1998), "Distribution and Mobility of Wealth of Nations", European Economic Review 42, 1269-1293.

Quah, D. (1993a), "Galton's Fallacy and Tests of the Convergence Hypothesis", Scandinavian Journal of Economics 95, 427-443.

Quah, D. (1993b), "Empirical Cross-Section Dynamics in Economic Growth", European Economic Review 37, 426-434.

Quah, D. (1996a), "Twin Peaks: Growth and Convergence in Models of Distribution Dynamics", Economic Journal 106, 1045-1055.

Quah, D. (1996b), "Empirics for Economic Growth and Convergence", European Economic Review 40, 1353-1375.

Quah, D. (1997), "Empirics for Growth and Distribution: Stratification, Polarization, and Convergence Clubs", Journal of Economic Growth 2, 27-59.

Raurich-Puigdevall, X. (2000), "Global Indeterminacy in an Endogenous-Growth Model with Public Capital", Journal of Economics 71, 255-280.

Silverman, B.W. (1986), Density Estimation for Statistics and Data Analysis, London: Chapman\&Hall.

Summers, R., and A. Heston (1991), "The Penn World Table (Mark 5): An Expanded Set of International Comparisons 1950-1988", Quarterly Journal of Economics 106, 327-368.

Wand, M.P., Jones, M.C. (1995), Kernel Smoothing, London: Chapman\&Hall. 